\title{
Relativistic Coulomb excitation of the giant dipole resonance in nuclei: How to calculate transition probabilities without invoking the Liénard-Wiechert relativistic scalar and vector potentials
}

\author{
C. H. Dasso and M. Gallardo \\ Departamento de Física Atómica, Molecular y Nuclear Facultad de Física, Apartado 1065, E-41080 Sevilla, Spain
}

(Received 4 August 2005; published 27 January 2006)

\begin{abstract}
The conclusions extracted from a recent study of the excitation of giant dipole resonances in nuclei at relativistic bombarding energies open the way for a further simplification of the problem. It consists in the elimination of the relativistic scalar and vector electromagnetic potentials and the familiar numerical difficulties associated with their presence in the calculation scheme. The inherent advantage of a reformulation of the problem of relativistic Coulomb excitation of giant dipole resonances along these lines is discussed.
\end{abstract}

DOI: 10.1103/PhysRevC.73.014907

PACS number(s): 21.60.Jz

\section{INTRODUCTION}

In a recent article [1] it was shown how it is possible to calculate accurate probabilities of excitation of the giant dipole resonance at the one- and two-phonon levels (GDR and DGDR, respectively) in very simple terms. A key conclusion of this reference was that the results obtained following the standard formalism of Winther and Alder [2,3] are-for those partial waves where they can be considered reliable-much less dependent on the specific structural details provided by a microscopic description of the collective mode than previously assumed.

In fact, for bombarding energies up to at least 5-10 GeV per nucleon and for situations that are compatible with a truncation of the model space at the level of the DGDR it is not compelling to have an elaborate picture of the distribution of charges and currents within the nucleus. The relevant information is contained in their lowest moments, namely the position of the center of charge and its velocity. A variety of structural models may share these features and this realization makes the investment of a significant effort in this direction rather impractical. For the concrete task of understanding the main features exhibited by the data obtained within present experimental conditions the simplest, familiar model of an oscillation of the proton density against the neutron density does suffice. Let us here recall that the basic parameters that are necessary to implement such picture are inferred from the known excitation energy of the mode and the mass and charge of the nucleus in question.

With this backround in mind we propose in this contribution to go a step further and eliminate from the calculation scheme of Ref. [1] the presence of the scalar Liénard-Wiechert potential for a pointlike projectile [4]

$$
\Phi(x, y, z, t)=\frac{\gamma Z_{p} e}{\sqrt{(x-b)^{2}+y^{2}+\gamma^{2}\left(z-v_{p} t\right)^{2}}},
$$

and, even more desiderably, its vector counterpart

$$
\vec{A}=\frac{\vec{v}_{P}}{c} \Phi .
$$

To this end we suggest to cast the problem in the reference system of the projectile, where the electric and magnetic fields in the whole space and at any instant of time are simply

$$
\vec{E}(\vec{r})=\frac{Z_{P} e}{r^{2}} \frac{\vec{r}}{r}, \quad \vec{B}(\vec{r})=0,
$$

that is, the same expressions that are normally used at nonrelativistic energies.

The price to be paid for such simplicity and the absence of vector potentials in the problem is that we must, of course, transform the restoring forces associated with the giant dipole resonance from a system in which the "spring" is at rest to another in which it moves with a speed $v \approx c$. This, as we shall see, is not that difficult to do. We would like to stress, however, that the reason for posing this problem is not entirely "academic." Notice that if one manages to incorporate successfully such transformation laws in the formalism it should be possible to compute the excitation probability of the mode via any other field whose expression in a system at rest is known.

Let us expand on the previous statement. The electromagnetic potentials are specifically easy to transform between relativistically moving systems of reference because the combination $(\vec{A}, \Phi)$ forms a four-vector whose change is governed by the four-by-four Lorentz transformation matrix. In fact, the Liénard-Wiechert expressions quoted above are no more than the Lorentz-transformed version of the electric and magnetic potentials generated by a point charge at rest. It is well known, however, that the transformation law for practically any other interaction is not that straightforward to obtain. It is because of this recurrent difficulty that it appears promising to incorporate in the formalism, once and for all, the transformation properties of the intrinsic forces that account for the response of the giant dipole resonance. Upon a successful completion of this program one would be able to test the effects of a variety of excitation couplings (for instance, nuclear) without having to worry about their properties under Lorentz transformations.

In this contribution we set out to explore the practical implementation of these ideas. In Sec. II we work out the formal aspects of solving the problem of excitation of the GDR as seen from the frame of reference of the projectile. The problem of the target recoil, already treated in detail in Ref. [1], is briefly reviewed in Sec. III. A comparison 


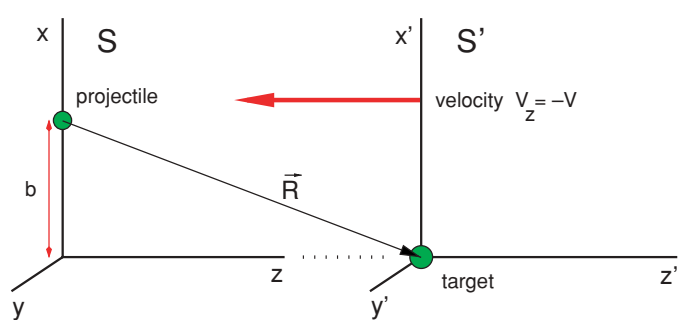

FIG. 1. (Color online) Reference frames used in the text. The projectile frame is $S$ and the target frame (the nucleus whose giant dipole mode is excited) is $S^{\prime}$. The latter moves toward the left in the $z$ direction with a velocity $v$ comparable to the speed of light. The origin of the two systems coincide for $t=0$ and the target-projectile relative coordinate $\vec{R}$ in the system $S$ has components $\left(R_{x}, R_{y}, R_{z}\right)=$ $(-b, 0,-v t)$, where $b$ is the impact parameter.

of the results obtained with the procedures presented in the previous Sec. with results extracted following the conventional formalism of Alder and Winther is the subject of Sec. IV. A summary of the article and some closing remarks are left for Sec. V.

\section{FORMALISM}

Because in relativistic heavy-ion collisions the excitation energies that enter into play $(\approx 10-20 \mathrm{MeV})$ are much smaller than the bombarding energies, we can consider the relative motion between projectile and target to be uniform and assume that the classical trajectories are well approximated by straight lines. Without loss of generality we take that direction to be along the $z$ axis. The problem we intend to approach is illustrated in Fig. 1. Here we see two systems of reference, $S$ and $S^{\prime}$, which move with respect to each other at a very large speed $\vec{v}$. The system $S$ is chosen so that the projectile is always at rest in the position $(b, 0,0)$, where $b$ plays the role of the classical impact parameter. It is in this frame of reference that we would like to write down the equations of motion that describe the externally driven oscillation of protons against neutrons in the target. We exploit in this section the same assumption made at the beginning of Ref. [1], namely that the center of mass of the proton and neutron distributions - i.e., the original "target"-remains at rest at the coordinates $\left(x^{\prime}, y^{\prime}, z^{\prime}\right) \equiv(0,0,0)$, the origin of system $S^{\prime}$. We verify in Sec. III that, although a priori unjustified, the use of this no-recoil approximation does not affect the conclusions of the analysis in any significant way. The time scales are chosen so that the $x$ axes in $S$ and $S^{\prime}$ overlap at $t=t^{\prime}=0$.

Under the conditions specified above, the coordinates in $S$ and $S^{\prime}$ are connected by the following:

$$
\begin{aligned}
x^{\prime} & =x, \\
y^{\prime} & =y, \\
z^{\prime} & =\gamma(z+v t), \\
t^{\prime} & =\gamma\left(t+\frac{v z}{c^{2}}\right),
\end{aligned}
$$

where $v$ is positive and the factor $\gamma$ associated with the Lorentz transformation between the two reference systems is

$$
\gamma=1 / \sqrt{1-(v / c)^{2}}
$$

The set of equations (4) can be supplemented with the one relating the spacelike velocities in the two systems, namely

$$
\begin{aligned}
\dot{x}^{\prime} & =\frac{\dot{x}}{\gamma\left(1+\dot{z} v / c^{2}\right)}, \\
\dot{y}^{\prime} & =\frac{\dot{y}}{\gamma\left(1+\dot{z} v / c^{2}\right)}, \\
\dot{z}^{\prime} & =\frac{\dot{z}+v}{\left(1+\dot{z} v / c^{2}\right)} .
\end{aligned}
$$

It is useful to recall a couple of formal relationships involving the $\gamma$ factors for the motion of particles in $S$ and $S^{\prime}$ (important at relativistic speeds in either system). We use them, below, to arrive at our final results. Calling $u^{2}=\left(\dot{x}^{2}+\dot{y}^{2}+\dot{z}^{2}\right)$ and $u^{\prime 2}=\left(\dot{x}^{\prime 2}+\dot{y}^{\prime 2}+\dot{z}^{\prime 2}\right)$ we introduce

$$
\begin{aligned}
\tilde{\gamma} & =1 / \sqrt{1-(u / c)^{2}}, \\
\tilde{\gamma}^{\prime} & =1 / \sqrt{1-\left(u^{\prime} / c\right)^{2}} .
\end{aligned}
$$

With these definitions it is possible to show that the three quantities $\gamma, \tilde{\gamma}$, and $\tilde{\gamma}^{\prime}$ satisfy

$$
\begin{aligned}
\gamma \tilde{\gamma} & =\tilde{\gamma}^{\prime} /\left(1+\dot{z} v / c^{2}\right), \\
\gamma \tilde{\gamma}^{\prime} & =\tilde{\gamma} /\left(1-\dot{z}^{\prime} v / c^{2}\right), \\
\gamma^{2} & =\frac{1}{\left(1+\dot{z} v / c^{2}\right)\left(1-\dot{z}^{\prime} v / c^{2}\right)} .
\end{aligned}
$$

We can ignore from now on equations involving one of the spatial orientations because the motion is constrained to take place on the $[x, z]$ (or $\left[x^{\prime}, z^{\prime}\right]$ ) plane and $y=y^{\prime}=0$ throughout. Keeping this in mind, we write the spacelike components of the force acting at the end of the elongated spring in the system $S^{\prime}$. They are as follows:

$$
\begin{aligned}
& {F_{x^{\prime}}^{\prime H O}}^{H O}=-C x^{\prime}, \\
& {F_{y^{\prime}}^{\prime H O}}^{{ }^{\prime}}=0, \\
& {F_{z^{\prime}}^{\prime H O}}^{{ }^{H O}}=-C z^{\prime} .
\end{aligned}
$$

Here we use the same notation as in Ref. [1]. The value of the restoring force parameter $C=D(\hbar \omega)^{2}$ is derived from the excitation energy $\hbar \omega$ of the mode and the reduced mass $D=$ $\left(Z_{T} N_{T} / A_{T}\right) m$, where $m$ is a nucleon mass. Starting from these expressions we construct the components of Minkowski's fourvector force in $S^{\prime}[8]$,

$$
\begin{aligned}
K_{1}^{\prime} & =-\tilde{\gamma}^{\prime} C x^{\prime}, \\
K_{2}^{\prime} & =0, \\
K_{3}^{\prime} & =-\tilde{\gamma}^{\prime} C z^{\prime}, \\
K_{4}^{\prime} & =-\frac{i}{c} \tilde{\gamma}^{\prime} C\left(x^{\prime} \dot{x}^{\prime}+z^{\prime} \dot{z}^{\prime}\right),
\end{aligned}
$$


which, Lorentz-transformed into $S$, yield

$$
\begin{aligned}
K_{1}= & -\tilde{\gamma}^{\prime} C x, \\
K_{2}= & 0, \\
K_{3}= & -C \gamma \tilde{\gamma}^{\prime}\left\{\gamma(z+v t)-\frac{v}{c^{2}}\left[x \frac{\dot{x}}{\gamma\left(1+\dot{z} v / c^{2}\right)}\right.\right. \\
& \left.\left.+\gamma(z+v t) \frac{\dot{z}+v}{\left(1+\dot{z} v / c^{2}\right)}\right]\right\}, \\
K_{4}= & -i C \gamma \tilde{\gamma}^{\prime}\left\{\frac{v}{c} \gamma(z+v t)+\left[x \frac{\dot{x}}{\gamma\left(1+\dot{z} v / c^{2}\right)}\right.\right. \\
& \left.\left.+\gamma(z+v t) \frac{\dot{z}+v}{\left(1+\dot{z} v / c^{2}\right)}\right]\right\} .
\end{aligned}
$$

From the previous expressions it is easy to identify the intrinsic restoring forces that should be used to construct the relativistic equations of motion for the collective variable $(x, y, z)$ in the system of coordinates $S$ and follow its evolution with respect to the variable $t$,

$$
\begin{aligned}
& F_{x}^{H O}=-C \gamma\left(1+\dot{z} v / c^{2}\right) x, \\
& F_{y}^{H^{O}}=0, \\
& F_{z}^{H O}=-C \gamma(z+v t)+C \gamma \frac{v}{c^{2}} x \dot{x} .
\end{aligned}
$$

Notice the word relativistic in the previous paragraph. It is quite clear that typical velocities in the system $S$ will be close to the speed of light and therefore the classical (meaning in this context "not quantal") equations we need to solve are Einstein's set

$$
\begin{aligned}
& \frac{d}{d t}\left[\frac{D \dot{x}}{\sqrt{1-\left(\dot{x}^{2}+\dot{z}^{2}\right) / c^{2}}}\right]=F_{x}=F_{x}^{H O}+\frac{Z_{P} Z_{T} e^{2} \delta}{R^{2}}\left(\frac{b}{R}\right), \\
& \frac{d}{d t}\left[\frac{D \dot{z}}{\sqrt{1-\left(\dot{x}^{2}+\dot{z}^{2}\right) / c^{2}}}\right]=F_{z}=F_{z}^{H O}+\frac{Z_{P} Z_{T} e^{2} \delta}{R^{2}}\left(\frac{v t}{R}\right) .
\end{aligned}
$$

To define the components of the total force $F_{x}, F_{z}$ we have added to the restoring force [Eq. (12)] the (now trivial) contribution from the Coulomb interaction. Within the dipole approximation one can use the value of the field at any point in the neighborhood of the origin of $S^{\prime}$ and we specifically take $R=\sqrt{b^{2}+v^{2} t^{2}}$. Notice the factor $\delta=N_{T} /\left(N_{T}+Z_{T}\right)$ in the expression of the electric force. It appears (cf. Ref. [1]) because we set up here equations of motion for the collective variable $\vec{r}=(x, y, z)$ and ignore the overall acceleration of the center of mass of the target. It should not enter in the formulation (and in fact it does not) if one integrates separately for the proton and neutron components, as it is later done in Sec. III.
The expressions (13) are not yet cast in a convenient form to be solved by standard integration methods. For this purpose we need to isolate the second time derivatives of $x, z$. Introducing the auxiliary quantities

$$
\begin{aligned}
\mathcal{A}(\dot{z}) & =1-\frac{\dot{z}^{2}}{c^{2}}, \\
\mathcal{B}(\dot{x}, \dot{z}) & =\frac{\dot{x} \dot{z}}{c^{2}}, \\
\mathcal{C}(\dot{x}) & =1-\frac{\dot{x}^{2}}{c^{2}},
\end{aligned}
$$

we arrive to a very compact set of time-dependent, first-order coupled differential equations in the variables $x, z, \dot{x}, \dot{z}$ to be numerically propagated from their initial values, namely

$$
\begin{aligned}
\frac{d x}{d t} & =\dot{x} \\
\frac{d z}{d t} & =\dot{z} \\
\frac{d \dot{x}}{d t} & =\frac{1}{D \tilde{\gamma}^{3}} \frac{F_{x} \mathcal{C}-F_{z} \mathcal{B}}{\mathcal{A C}-\mathcal{B}^{2}}, \\
\frac{d \dot{z}}{d t} & =\frac{1}{D \tilde{\gamma}^{3}} \frac{F_{z} \mathcal{A}-F_{x} \mathcal{B}}{\mathcal{A} C-\mathcal{B}^{2}} .
\end{aligned}
$$

The final excitation energy of the dipole mode is calculated after integration of [Eq. (15)] and-dividing by $\hbar \omega-$ converted into an average number of phonons $N_{\infty}$. In turn, this number is converted into excitation probabilities for the ground state and the one- and two-phonon levels, just as it was done in Ref. [1] for situations where $N_{\infty} \ll 1$.

A computer code called REVRCE has been written to implement this prescription. To illustrate the outmost simplicity of this approach we show in Fig. 2 an overview of the complete listing of this Fortran program. The inset identifies the only piece of the program where the Coulomb interaction appears [cf. Eq. (13)].

We compare in Sec. IV the results obtained using the REVRCE code with state-of-the-art calculations following the formulation of Alder and Winther.

\section{TARGET RECOIL}

In Ref. [1] it was discussed in detail the impact of the common practice of calculating the excitation of the dipole mode in a coordinate system attached to the target. Being this a nucleus with a net positive charge it is actually accelerated during the collision process and the presence of inertial forces should not be ignored without a proper investigation. It is not necessary here to adapt the entire line of arguments to the present situation. Rather, we limit ourselves to quote the equations of motion that should be solved in system $S$ to follow the separate motion of the charged and neutral components of the nuclear density.

In terms of the two independent collective variables $\vec{r}^{p}=\left(x^{p}, y^{p}, z^{p}\right) \quad\left(p\right.$ for protons) and $\vec{r}^{n}=\left(x^{n}, y^{n}, z^{n}\right)$ ( $n$ for neutrons) the set of equations, now eight in total, is 


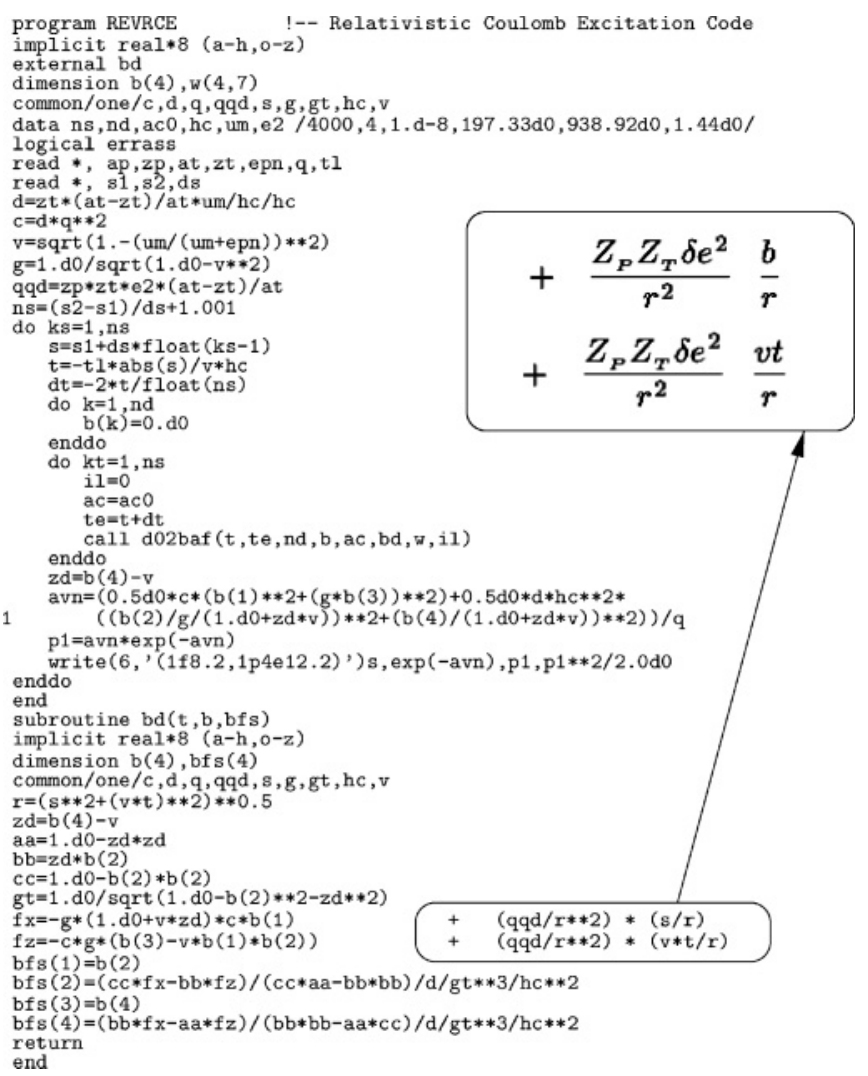

FIG. 2. An overview of the fortran program REVRCE that calculates the excitation probabilities for the GDR and the DGDR according to the formalism presented in Sec. II. The program is complete and self-contained with the exception of a single call to a standard integration routine D02BAF [9]. The boxes are included to draw attention to the only part of the program where the electromagnetic interaction enters in the equations of motion, assuming the simple form generated by a charge at rest in a neighborhood of the target (dipole approximation).

as follows:

$$
\begin{aligned}
\frac{d x^{p}}{d t} & =\dot{x}^{p} \\
\frac{d z^{p}}{d t} & =\dot{z}^{p} \\
\frac{d \dot{x}^{p}}{d t} & =\frac{1}{Z_{T} m \tilde{\gamma}_{p}^{3}} \frac{F_{x}^{p} \mathcal{C}^{p}-F_{z}^{p} \mathcal{B}^{p}}{\mathcal{A}^{p} \mathcal{C}^{p}-\mathcal{B}^{p}} \\
\frac{d \dot{z}^{p}}{d t} & =\frac{1}{Z_{T} m \tilde{\gamma}_{p}^{3}} \frac{F_{z}^{p} \mathcal{A}^{p}-F_{x}^{p} \mathcal{B}^{p}}{\mathcal{A}^{p} \mathcal{C}^{p}-\mathcal{B}^{p}}
\end{aligned}
$$

and

$$
\begin{aligned}
\frac{d x^{n}}{d t} & =\dot{x}^{n}, \\
\frac{d z^{n}}{d t} & =\dot{z}^{n}, \\
\frac{d \dot{x}^{n}}{d t} & =\frac{1}{N_{T} m \tilde{\gamma}_{n}^{3}} \frac{F_{x}^{n} \mathcal{C}^{n}-F_{z}^{n} \mathcal{B}}{\mathcal{A}^{n} \mathcal{C}^{n}-\mathcal{B}^{n 2}}, \\
\frac{d \dot{z}^{n}}{d t} & =\frac{1}{N_{T} m \tilde{\gamma}_{n}^{3}} \frac{F_{z}^{n} \mathcal{A}^{n}-F_{x}^{n} \mathcal{B}^{n}}{\mathcal{A}^{n} \mathcal{C}^{n}-\mathcal{B}^{n 2}} .
\end{aligned}
$$

In these expressions

$$
\begin{aligned}
\mathcal{A}^{p}\left(\dot{z}^{p}\right) & =1-\frac{\dot{z}^{p 2}}{c^{2}}, \\
\mathcal{B}^{p}\left(\dot{x}^{p}, \dot{z}^{p}\right) & =\frac{\dot{x}^{p} \dot{z}^{p}}{c^{2}}, \\
\mathcal{C}^{p}\left(\dot{x}^{p}\right) & =1-\frac{\dot{x}^{p 2}}{c^{2}}, \\
\mathcal{A}^{n}\left(\dot{z}^{n}\right) & =1-\frac{\dot{z}^{n 2}}{c^{2}}, \\
\mathcal{B}^{n}\left(\dot{x}^{n}, \dot{z}^{n}\right) & =\frac{\dot{x}^{n} \dot{z}^{n}}{c^{2}}, \\
\mathcal{C}^{n}\left(\dot{x}^{n}\right) & =1-\frac{\dot{x}^{n 2}}{c^{2}} .
\end{aligned}
$$

The forces acting on the charged and neutral components are, respectively

$$
\begin{aligned}
& F_{x}^{p}=-C \gamma\left(1+\dot{z}^{p} v / c^{2}\right)\left(x^{p}-x^{n}\right)+\frac{Z_{P} Z_{T} e^{2}}{R^{2}}\left(\frac{b}{R}\right), \\
& F_{z}^{p}=-C \gamma\left(z^{p}-z^{n}\right)+C \gamma \frac{v}{c^{2}}\left(x^{p}-x^{n}\right) \dot{x}^{p}+\frac{Z_{P} Z_{T} e^{2}}{R^{2}}\left(\frac{v t}{R}\right),
\end{aligned}
$$

and

$$
\begin{aligned}
& F_{x}^{n}=-C \gamma\left(1+\dot{z}^{n} v / c^{2}\right)\left(x^{n}-x^{p}\right), \\
& F_{z}^{n}=-C \gamma\left(z^{n}-z^{p}\right)+C \gamma \frac{v}{c^{2}}\left(x^{n}-x^{p}\right) \dot{x}^{n} .
\end{aligned}
$$

Notice that the factor $\delta$ does not-as anticipated-scale the Coulomb interaction term acting now only on the charged density.

A computer program called REVRCE2c (also very simple) has been written to implement this prescription. We compare in the following section the results obtained using this code with those of calculations performed acording to the standard approach.

\section{COMPARISON WITH THE STANDARD FORMALISM}

In this brief section we compare results for the probability of excitation of the giant dipole resonance of ${ }^{40} \mathrm{Ca}$ in the reaction ${ }^{208} \mathrm{~Pb}+{ }^{40} \mathrm{Ca}$ at the relativistic bombarding energies of 500 , 1000 , and $4000 \mathrm{MeV}$ per nucleon. On the one hand, we have the predictions of the codes REVRCE and REVRCE2C according to the prescriptions discussed in Sec. II and III, respectively. On the other hand, the results of Bayman and Zardi obtained with high computational precision following the approach of Alder and Winther [5,6].

Results for a large number of impact parameters are collected in Fig. 3. The lower limit in the range of partial waves represented in the drawing is determined-as it was already mentioned in Ref. [1] — excluding from the set those values that are incompatible with a "safe" truncation of the model Hamiltonian at the two-phonon level. This is, equivalently, the regime of validity of perturbation theory where $P_{0} \approx 1$ throughout. On the large impact-paramater side 


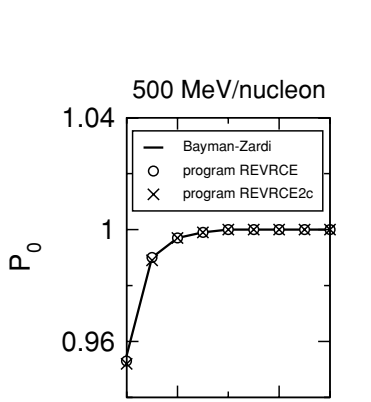

${ }^{40} \mathrm{Ca}+{ }^{208} \mathrm{~Pb}$

$1000 \mathrm{MeV} /$ nucleon
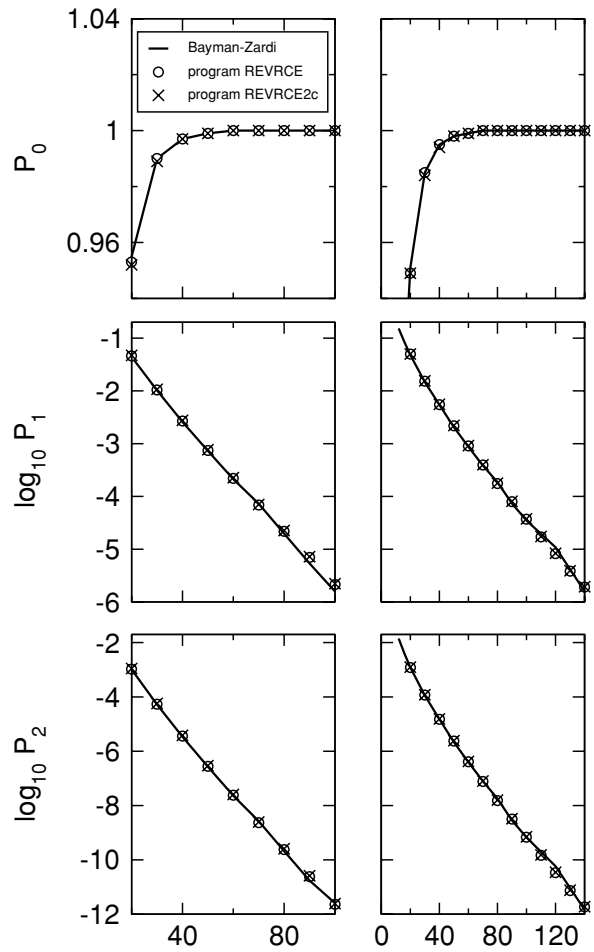

Impact Parameter (fm)
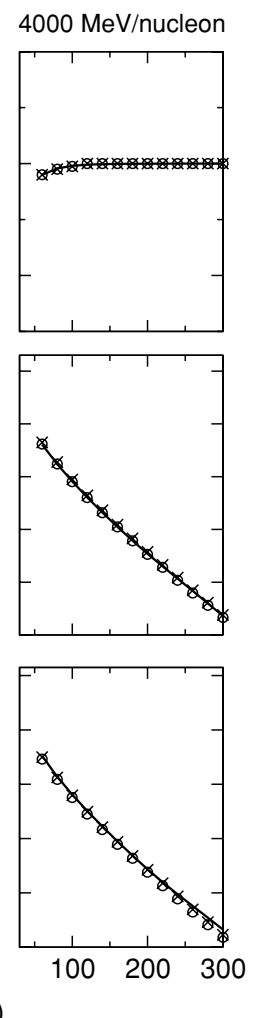

FIG. 3. Probabilities for the excitation of the GDR $\left(P_{1}\right)$ and the double GDR $\left(P_{2}\right)$ in ${ }^{40} \mathrm{Ca}$ in the reaction ${ }^{208} \mathrm{~Pb}+{ }^{40} \mathrm{Ca}$ at bombarding energies of 500,1000, and $4000 \mathrm{MeV}$ per nucleon. The energy of the GDR was assumed to be $11.6 \mathrm{MeV}$. In the first row the probability $P_{0}$ for remaining in the elastic channel is also shown. The three sets of results give the predictions obtained from the simplest classical model (REVRCE), with the inclusion of target recoil (REVRCE2c) and in the conventional approach (Bayman-Zardi).

the practical limit is set by the reliability of the traditional calculations, whose accuracy is, naturally, much more difficult to mantain.

We can see that the different methods yield, in all circumstances, very similar results. The agreement obtained with the codes REVRCE and REVRCE2c should not come as a surprise, though, because the validity of the no-recoil approximation had been established in Ref. [1] and is not affected by a reformulation of the problem through Lorentz transformations.

\section{SUMMARY AND CONCLUSIONS}

In this manuscript we set out to verify that an alternative approach to the problem of relativistic Coulomb excitation of giant dipole resonances is possible. Our aim was the evaluation of transition probabilities at relativistic bombarding energies avoiding the introduction in the calculation scheme of the Liénard-Wiechert potentials.

Such project could have not been contemplated without having available the results previously obtained in Ref. [1]. There we learned that - within the range of impact parameters where the active reaction channels involve only the ground state and the first two excited states - the intrinsic motion can be satisfactorily modelled by a collective oscillation of the charged and neutral components of the total nuclear density against each other. The problem is then technically formulated in terms of a macroscopic variable $\vec{r}$ that represents the displacement of centers of each distribution density with respect to their equilibrium position. This is, of course, one of the oldest visualizations of the intrinsic motion associated with a giant dipole resonance in nuclei. What was not obvious, perhaps, is that such simple scheme could yield accurate excitation probabilities and handle so well the current trend to push bombading energies up into the relativistic regime.

Describing the intrinsic mode in terms of an harmonic vibration it has been relatively straightforward to recast the solution of the problem through a new set of equations of motion where the electric interaction enters in the simplest possible form indicated in Eqs. (13) and (19). The practical advantage of the formulation can be appreciated in the remarkable simplicity of the calculation tool shown in Fig. 2 and the excellent agreements displayed in Fig. 3. Notice, also, that in the right-hand sides of Eqs. (13) and (19) one could easily add additional terms to consider the effect of other types of couplings.

In the case of the GDR the analogy with a classical three-dimensional spring can be exploited to its fullest extent. Let us mention, however, that there are well-established techniques that employ a similar semiclassical language for handling the excitation of collective harmonic vibrations of other multipolarities in reactions with heavy ions [7] that can be a source of inspiration for future developments.

\section{ACKNOWLEDGMENTS}

Support is acknowledged from the Ministry of Educaction and Science under project numbers FIS2005-01105 and FPA2005-04460.
[1] C. H. Dasso, M. Gallardo, H. M. Sofia, and A. Vitturi, Phys. Rev. C 70, 044903 (2004).

[2] A. Winther and K. Alder, Nucl. Phys. A319, 518 (1979).

[3] K. Alder and A. Winther, Electromagnetic Excitation (North Holland, Amsterdam, 1975).

[4] The espressions correspond to the electromagnetic potentials generated by a classical motion of the projectile along the $z$ axis with impact parameter $b$ and a velocity $\vec{v}_{P}$.

[5] B. F. Bayman and F. Zardi, Phys. Rev. C 68, 014905 (2003).
[6] B. F. Bayman and F. Zardi, Phys. Rev. C 59, 2189 (1999).

[7] R. Broglia, C. H. Dasso, and A. Winther, in Proceedings of the International School of Physics "Enrico Fermi” (North Holland, Amsterdam, 1981), Course LXXVII, p. 327.

[8] H. Goldstein, Classical Mechanics (Addison-Wesley, Reading, MA, 1950).

[9] NAG Fortran Library, The Numerical Algorithms Group Ltd, Oxford. 